\title{
Design and development of a coaxial cryogenic probe for precision measurements of the quantum Hall effect in the $A C$ regime
}

\author{
Martina Marzano ${ }^{1}$, Ngoc Thanh Mai Tran ${ }^{1,2}$, Vincenzo D'Elia ${ }^{1}$, Danilo Serazio ${ }^{1}$, Emanuele Enrico ${ }^{1}$, \\ Massimo Ortolano ${ }^{2,1}$, Klaus Pierz ${ }^{3}$, Jan Kučera ${ }^{4}$, Luca Callegaro ${ }^{1}$ \\ 1 INRIM Istituto Nazionale di Ricerca Metrologica, Strada delle Cacce 91, 10135, Turin, Italy \\ 2 Politecnico di Torino, Corso Duca degli Abruzzi 24, 10129, Turin, Italy \\ 3 PTB Physikalisch-Technische Bundesanstalt, Bundesallee 100, 38116 Braunschweig, Germany \\ ${ }^{4}$ CMI Czech Metrology Institute, Okružní 31, 63800 Brno, Czech Republic
}

\section{ABSTRACT}

The quantum Hall effect is the basis for the realisation of the resistance and impedance units in the International System of units since 2019. This paper describes a cryogenic probe that allows to set graphene Hall devices in quantisation conditions in a helium bath (4.2 $\mathrm{K}$ ) and magnetic fields up to $6 \mathrm{~T}$, to perform precision measurements in the $\mathrm{AC}$ regime with impedance bridges. The probe has a full coaxial wiring, isolated from the probe structure, and holds the device in a TO-8 socket. First, characterization experiments are reported on a GaAs device, showing quantisation at $5.5 \mathrm{~T}$. In the AC regime, multiple-series connections will be employed to minimize the residual error, quantified by electrical modelling of the probe.

\section{Section: RESEARCH PAPER}

Keywords: Quantum Hall effect; Metrology; Impedance; Graphene, Cryogenic probe

Citation: Martina Marzano, Ngoc Thanh Mai Tran, Vincenzo D'Elia, Danilo Serazio, Emanuele Enrico, Massimo Ortolano, Klaus Pierz, Jan Kucera, Luca Callegaro, Design and development of a coaxial cryogenic probe for precision measurements of the quantum Hall effect in the AC regime, Acta IMEKO, vol. 10, no. 2, article 5, June 2021, identifier: IMEKO-ACTA-10 (2021)-02-05

Section Editor: Ciro Spataro, University of Palermo, Italy

Received December 20, 2020; In final form April 26, 2021; Published June 2021

Copyright: This is an open-access article distributed under the terms of the Creative Commons Attribution 3.0 License, which permits unrestricted use, distribution, and reproduction in any medium, provided the original author and source are credited.

Funding: GIQS: Graphene Impedance Quantum Standard is a Joint Research Project, code 18SIB07, of the European Metrology Programme for Innovation and Research (EMPIR). This project received funding from the European Metrology Programme for Innovation and Research (EMPIR) co-financed by the Participating States and from the European Union's Horizon 2020 research and innovation programme.

Corresponding author: Martina Marzano, e-mail: m.marzano@inrim.it

\section{INTRODUCTION}

In the revised International System of Units (SI) the units of electrical impedance (ohm, henry, farad) can be realised from the quantised Hall resistance $R_{H}=R_{K} / i$, where $R_{K}=h / e^{2}=$ $25812.8074593045 \ldots \Omega$, the von Klitzing constant, is an exact value [1] and $i$ is a small integer (typically, $i=2$ ). The aim of the project GIQS: Graphene Impedance Quantum Standard (see Acknowledgments) is to develop and make available an affordable and easy-to-operate impedance standard exploiting the quantum Hall effect (QHE) in graphene.

Graphene devices are of strong interest for the realisation of electrical units since they display the QHE at lower magnetic fields (below $5 \mathrm{~T}$ ) and higher temperatures (several $\mathrm{K}$ ) than semiconductor devices, such as the well-established GaAs ones [2]-[5]. The operating conditions can thus be achieved with simpler and less expensive cryogenic systems. Nevertheless, the direct measurement of the quantised resistance in the $\mathrm{AC}$ regime requires careful considerations about device wiring and shielding [6]-[8], to minimise the effects of stray parameters which can alter the apparent resistance of the device from the quantised value [7].

To date, the direct traceability of capacitance to the QHE has been implemented with a coaxial transformer quadrature bridge, using two independent QHE devices in a twin probe [9]-[11]. The development of high-accuracy digital impedance bridges [12], [13] opens the possibility for simplified implementations. Among the goals of the GIQS project is the development of 

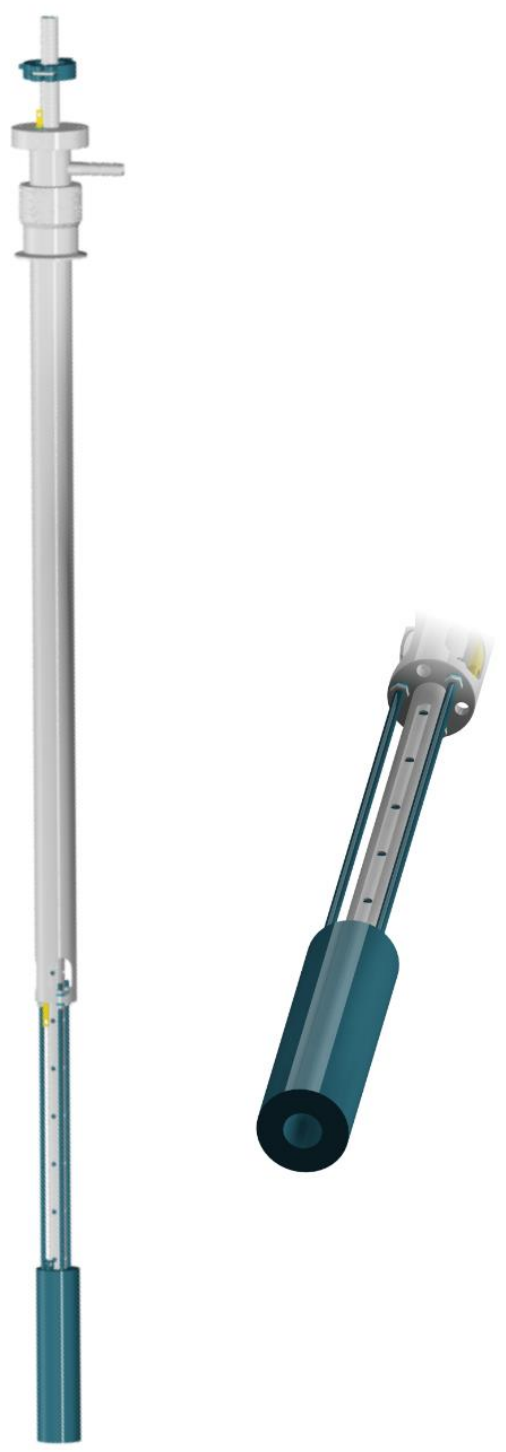

Figure 1. A computer rendering of the cryomagnetic probe assembly: (left) side view; (right) front view, showing the magnet bore.

impedance standards based on electronic fully-digital impedance bridges [8], [14]-[16] associated to individual QHE devices operating in a simple cryogenic environment.

The following describes the realisation of a cryogenic environment for QHE devices, which includes a coaxial cryogenic probe and a superconducting magnet of small size. The environment is suitable to perform QHE experiments at liquid helium temperature of $4.2 \mathrm{~K}$, and for an applied magnetic induction up to $6 \mathrm{~T}$, adequate for graphene QHE devices.

The probe will be employed with a new fully-digital coaxial impedance bridge developed in [16]. The bridge is optimised to perform $R C$ comparisons with a 1:1 magnitude ratio, with a comparison uncertainty around $10^{-7}$. In combination with the probe, it will allow the calibration of a capacitance standard in terms of $R_{H}$ and therefore the realisation of the unit of capacitance, the farad. The expected target relative uncertainty is $2 \times 10^{-7}$.

1 The probe frame was fabricated by Graphensic AB, Sweden, according to INRIM specifications.

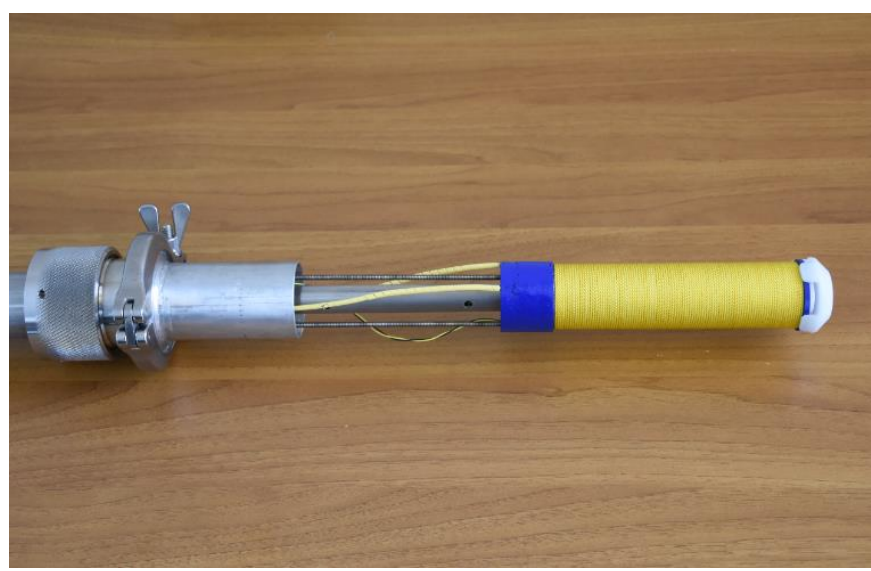

Figure 2. The probe cryomagnet, showing the yellow winding insulation. A header (white) was added to ease the insertion in the cryostat.

In the following, the probe is described and tested in the DC regime, using a novel GaAs sample that shows quantization plateaus for a magnetic induction (5.5 $\mathrm{T}$ for the $i=2$ plateau) lower than typical devices employed in metrology [7]. The results of the measurement are reported.

When employed in the AC regime, the parasitic parameters of the device, the sample holder and the probe will shift the apparent resistance, measured at the end of the coaxial wiring, with respect to the quantized value. The minimization of this shift with the implementation of the multiple-series connection scheme is discussed.

\section{THE PROBE}

A simplified diagram of the probe assembly ${ }^{1}$ is shown in Figure 1. The small size of the probe allows the insertion in a standard dewar having a $50 \mathrm{~mm}$ port, such as transport dewars.

\subsection{Superconducting magnet}

The probe supports a superconducting magnet (see Figure 2) that can reach a field up to $6 \mathrm{~T}$ at $4.2 \mathrm{~K}$ when energized with a DC current of approximately $65 \mathrm{~A}$. The field homogeneity is $0.1 \%$ over a $10 \mathrm{~mm}$ length. The magnet includes a superconducting thermal switch, which allows its operation in persistent mode.

\subsection{Probe insert}

The probe holds a sliding insert. The bottom side of the insert (Figure 3), which enters the magnet bore, mounts a TO-8 socket connected with nine coaxial wires ${ }^{2}$. Of these nine wires, eight are intended to be used on the current and voltage terminals of the device; the 9th wire is available for driving a section of the double shield (see below).

Samples can be mounted on special TO-8 sample holders (Figure 4) implementing a double-shielding technique [17] that minimises the frequency dependence of the quantised Hall resistance caused by unwanted stray capacitances. The socket is wired with the inner conductors of the coaxial cables; the outer conductors are connected to a common node very close to the sample holder.

The coaxial wiring (Figure 5) is terminated on a connection box at the top of the insert (Figure 6) with isolated British Post

${ }^{2}$ Lakeshore Ultra Miniature Coaxial Cable, type SC, Teflon insulation. The wire has an outer diameter of $1 \mathrm{~mm}$; the inner conductor has a series resistance per unit length of $0.282 \Omega \mathrm{m}^{-1}$ at room temperature; the inner to outer capacitance per unit length is $154 \mathrm{pF} \mathrm{m}^{-1}$. 


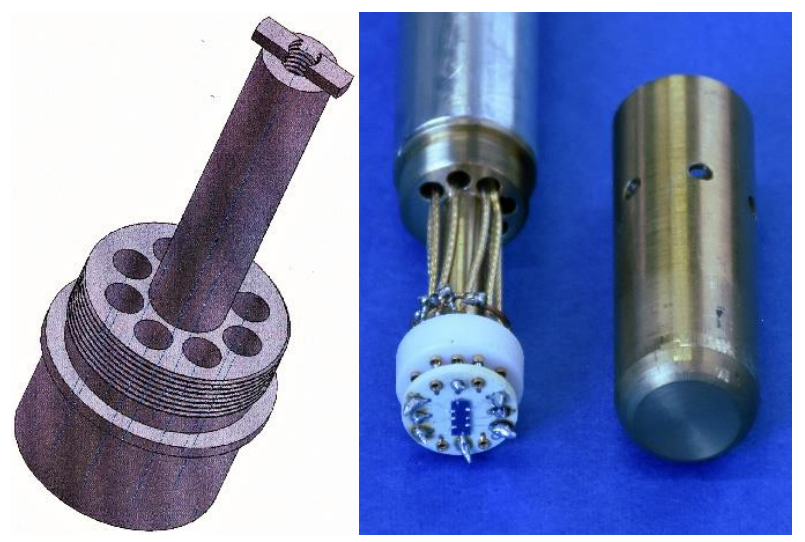

Figure 3. The bottom side of the insert, which slides into the cryomagnet bore. (left) Diagram of the drum that supports the sample holder and hosts the 9 coaxial connections. (right) Photo of the assembled probe: the brass shield (right) has been removed to show the TO-8 socket, which hosts a GaAs device on its TO- 8 holder, and the 9 coaxial electrical lines.

Office Multiple Unit Steerable Array (BPO MUSA) coaxial connectors. Often employed in impedance metrology, BPOMUSA connectors exhibit a good performance also in the DC regime. The whole coaxial network composed of the device and the wiring is completely isolated from the probe.

\subsection{Operation}

Figure 7 shows the probe in operation. It is inserted into a $60 \mathrm{~L}$ liquid helium dewar. The magnet is energised with a DC high-current power supply (Cryogenics PS 120A). A manual current-reversing switch allows to reverse the magnetic field polarity.

The superconducting switch in parallel with the magnet is driven by a DC laboratory power supply, which is turned off to activate the persistent mode of operation. The persistent mode maximises field stability and minimizes helium consumption, since the magnet wires are unloaded, and can be used when performing precision measurements on a QHE plateau.

\section{TESTING}

A test of the probe was made by performing measurement in the DC regime on a GaAs sample.

\subsection{GaAs sample}

The investigated sample, P151-24, was fabricated at the Physikalisch-Technische Bundesanstalt (PTB) facilities. Details of the process can be found in [18].

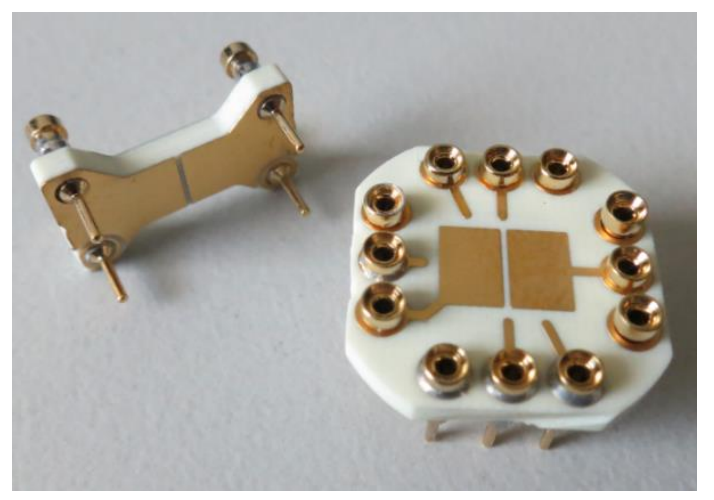

Figure 4. TO-8 sample holders, implementing the double-shielding technique [8], [17]. After the bonding of the quantum Hall device onto the holder (right), the shielding cap (left) slids into the socket.

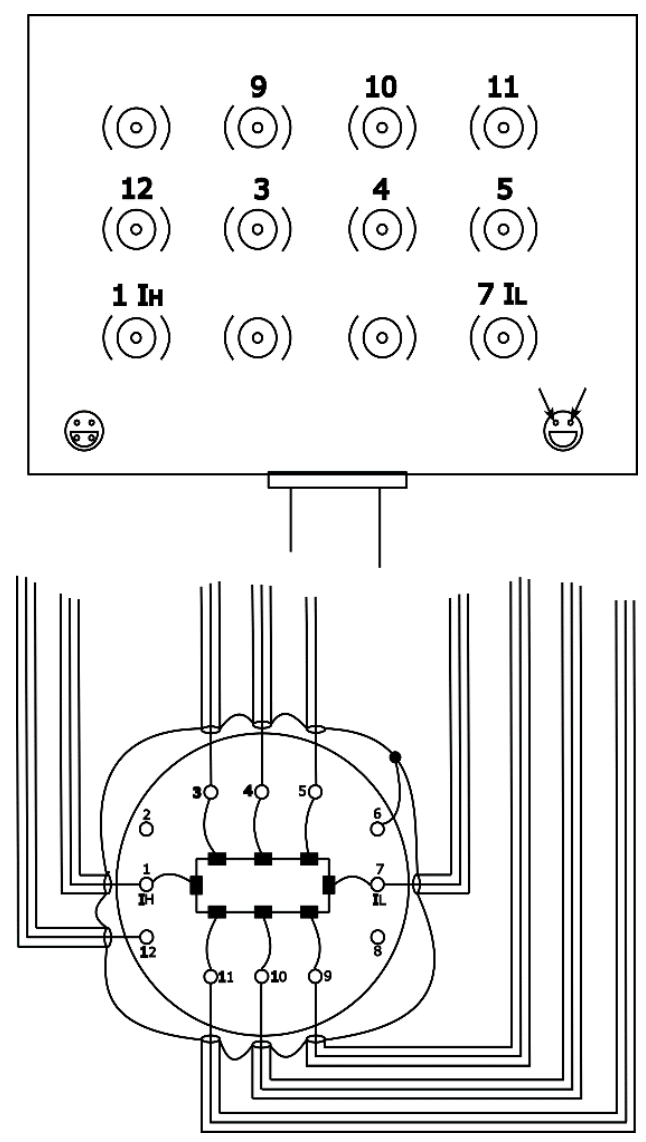

Figure 5. Schematic diagram of the coaxial connections of the probe. Nine coaxial connections are available, fully isolated from the probe metal bulk. The outer conductors of each line are joined together on the sample holder (pin 6).

P151 is a $500 \mu \mathrm{m}$ semi-insulating GaAs wafer on which a GaAs-AlGaAs heterostructure was grown by Molecular Beam Epitaxy (MBE). The wafer was cleaved in rectangles of $7 \mathrm{~mm} \times 3 \mathrm{~mm}$, and 8 contacts (two current and six voltage contacts) were made by tin ball annealing. The contacts have a resistance of about $10 \mathrm{~m} \Omega$.

P151 samples achieve a mobility of $58.5 \mathrm{~T}^{-1}$ and a carrier concentration in the two-dimensional electron gas $n=2.67 \times 10^{-15} \mathrm{~m}^{-2}$. This concentration corresponds to a $i=2$ quantum Hall plateau at the magnetic induction $B=n b / i e=5.5 \mathrm{~T}$, where $b$ is the Planck constant and $e$ the electron charge. Such magnetic induction can be compared with the typical induction required by ubiquitous LEP samples [19],

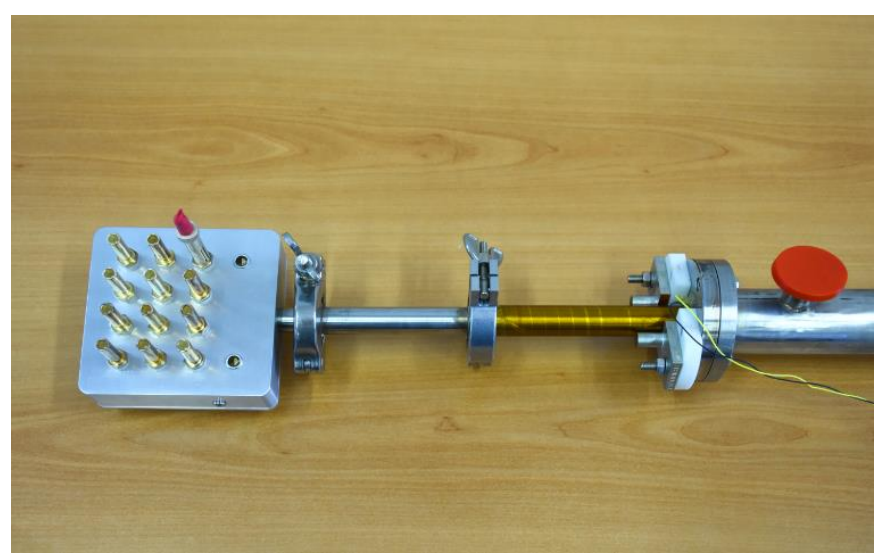

Figure 6. The probe connection box. 
of about $9 \mathrm{~T}$. Tests at $T=2.2 \mathrm{~K}$ and $I=39 \mu \mathrm{A}$ show full quantization to parts in $10^{\circ}$, and a critical current density of $6 \times 10^{-2} \mathrm{~A} \mathrm{~m}^{-1}(30 \mu \mathrm{A}$ over $0.5 \mathrm{~mm})$ at $T=1.2 \mathrm{~K}$ [18]. At $T=4.2 \mathrm{~K}, I=77 \mu \mathrm{A}$, the relative deviation from the exact quantization is about $-0.6 \times 10^{-6}$.

The sample is mounted on an unshielded TO- 8 holder with soldered Pt wires.

\subsection{Measurements}

The device is driven by a purpose-built, isolated and batteryoperated DC current source. The source can be manually operated to deliver the current values $I=0 \mu \mathrm{A}, \pm 5 \mu \mathrm{A}, \pm 20 \mu \mathrm{A}$, $\pm 50 \mu \mathrm{A}$ and $\pm 100 \mu \mathrm{A}$, and includes a fast protection circuit in case of device thermal runaway. The DC voltage on selected contacts is measured with a two-channel nanovoltmeter (Agilent 34420A).

Figure 8 reports the outcome of the experiment. Figure 8(a) is the graph of the Hall resistance $R_{H}(B)$ versus the applied magnetic field $B$, measured with a current $I=20 \mu \mathrm{A}$. Figure 8(b) shows the corresponding longitudinal resistance $R_{\mathrm{xx}}(B)$. Curves obtained with increasing and decreasing $B$ are shown; a little hysteresis, related to the sweep rate (about $0.6 \mathrm{~T} \mathrm{~min}^{-1}$ ) can be appreciated. Increasing the current up to $I=50 \mu \mathrm{A}$ leads to similar results (not reported).

In Figure 8(a) quantum Hall plateaux corresponding to filling factors $i=2$ and $i=4$ can be easily identified, and higher-index plateaux can be appreciated. Figure 8(b) shows the corresponding Shubnikov-de Haas oscillations. On the $i=2$ plateau, $R_{H}$ is flat over a range of about $0.2 \mathrm{~T}$; the corresponding $R_{x x}$ is lower than $50 \mathrm{~m} \Omega$. The outcome of the experiment is consistent with the expectations on a GaAs device.

\section{AC QUANTUM HALL EFFECT}

In the DC regime the quantum Hall resistance is defined as a four-terminal (4T) resistance [20]. If the 4T definition is applied (no current is drawn from the voltage terminals by the measurement setup) the cable errors are due to the wiring parasitic conductances, which can be made negligible with adequate isolation.

In the AC regime, the four terminal-pair (4TP) impedance standard definition is the most accurate $[20,21]$. 4TP impedance definition is however impractical for the quantum Hall resistance. Since the output impedance of the voltage terminals of a QHE device is of the order of $R_{H}$, the parasitic admittances of the cables give rise to very large errors at both the current and the voltage terminal-pairs. Attempts to solve the problem by triaxial connections and active guards were not completely successful [22].

The peculiarity of the quantized Hall state as a circuit element [23] allows to exploit the so-called multiple-series connections [24]. Such connections redefine the quantum Hall resistance as a twoterminal resistance (in the DC regime) or a two terminal-pair (2TP) impedance (in AC) by keeping the magnitude of the cable correction errors to very low values.

In the DC regime, the behaviour of multiple-series connections has been extensively considered [24], and dedicated modelling tools for the electrical analysis of the connections are available [25]-[27].

In the AC regime, multiple-series connections have been also implemented, although in a limited number of setups [3, 6-8]. The connections schematics are shown in Figure 9. For the case $n=1$, no multiple-series schematic is employed, and the device is simply connected as a $2 \mathrm{TP}$ impedance with two coaxial leads.

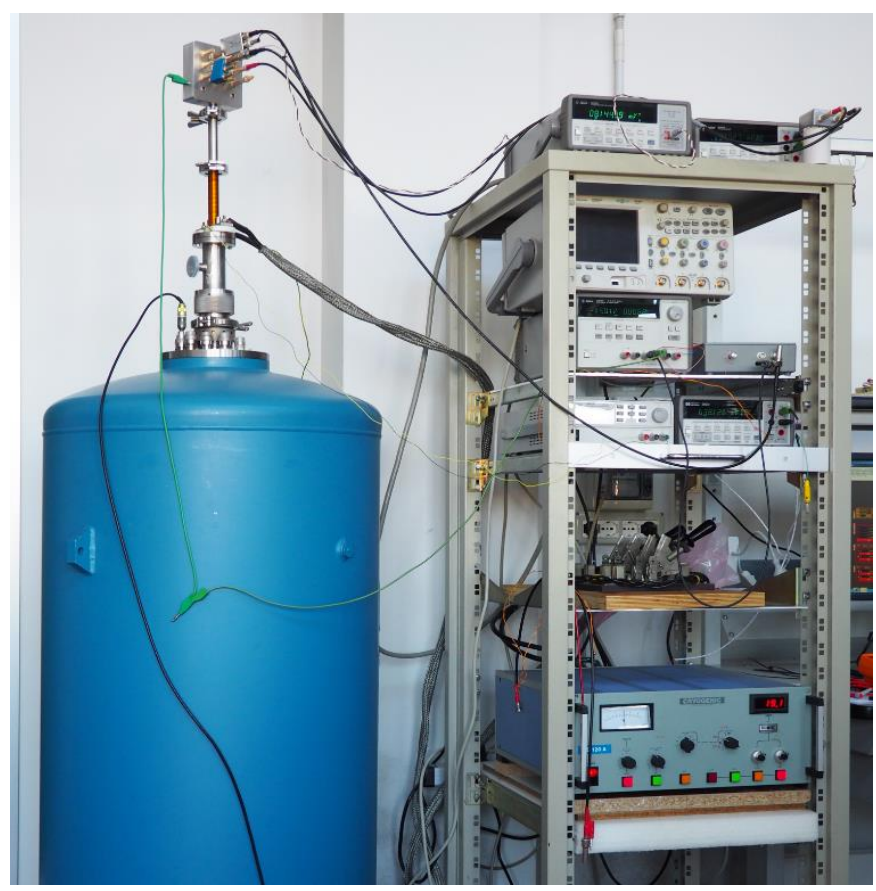

Figure 7. Overall view of the measurement setup for DC characterization. On the left, the liquid helium dewar, with the coaxial probe inserted. On the right, the rack of electronic instrumentation.

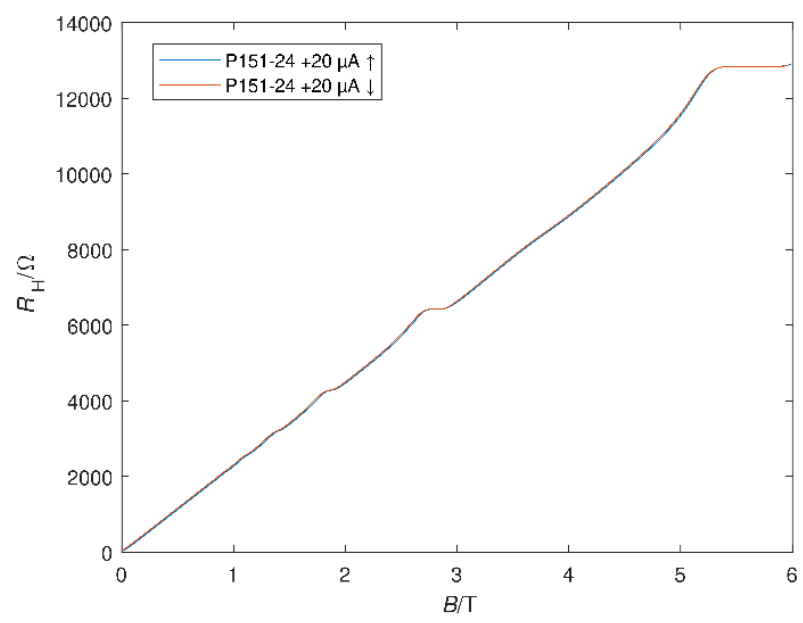

(a) $R_{\mathrm{H}}(B)$

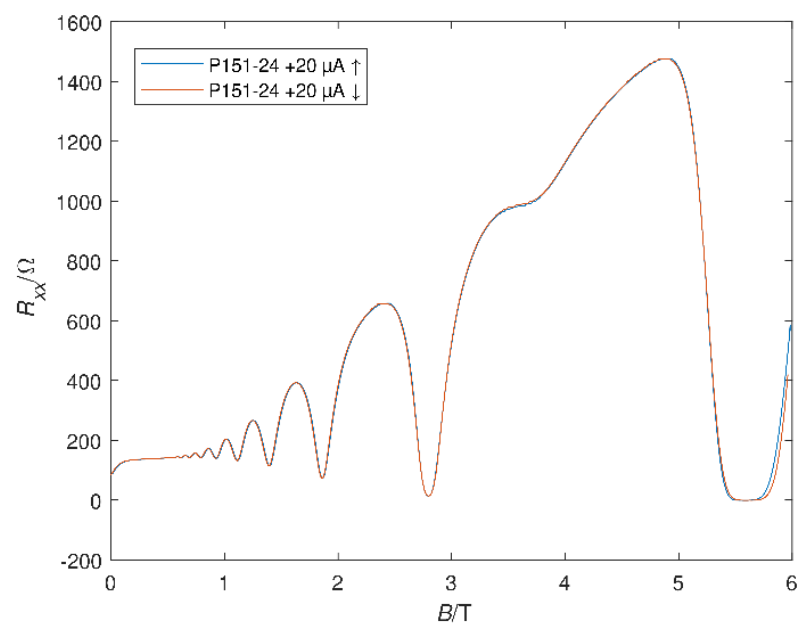

(b) $R_{\mathrm{xx}}(B)$

Figure 8. Plot of the measured values of $R_{\mathrm{H}}$ and $R_{\mathrm{xx}}$ versus the magnetic field $B$, for an applied current $I=20 \mu \mathrm{A}$. Blue line: increasing magnetic field. Red line: decreasing magnetic field. 
Table 1. Calculation of the real part of the relative error $\delta Z_{\mathrm{H}}$ occurring when measuring a QHE device by using the $n$-series connections given in Figure 9 , for the frequency $f=1541 \mathrm{~Hz}$. The calculation is given for two different contact resistance values.

\begin{tabular}{cccc}
\hline $\operatorname{Re}\left[\delta Z_{\mathrm{H}}\right]$ & $\boldsymbol{n}$ & $\boldsymbol{R}_{\mathrm{C}}=10 \mathrm{~m} \Omega$ & $\boldsymbol{R}_{\mathrm{C}}=10 \Omega$ \\
\hline 1 & $+1.0 \times 10^{-4}$ & $+1.7 \times 10^{-3}$ \\
2 & $-5.6 \times 10^{-9}$ & $+1.4 \times 10^{-6}$ \\
3 & $-1.1 \times 10^{-8}$ & $-9.5 \times 10^{-9}$ \\
\hline
\end{tabular}

The case $n=2$ and $n=3$ are called double series and triple series, respectively. Cases with $n>3$ can be conceived but are not analysed here.

The electrical modelling of multiple-series connections in the AC regime is much more complex than the DC case, and yet to be fully developed. Here, we apply an approximate model due to Schurr et al. [28]. As can be seen from Figure 10, each coaxial wiring is modelled as a T network with series impedance $Z_{\mathrm{w}}$ (mainly due to the series resistance of the inner conductor, which can be relatively high $-1 \Omega$ or greater - in cryogenic coaxial leads) and a parallel admittance $Y_{\mathrm{w}}$ (due to the cable capacitance and loss); a contact resistance $R_{C}$ models the bonding and the device junctions.

The quantity of interest to be estimated is the relative deviation $\delta Z_{\mathrm{H}}$ caused by the connections

$$
\delta Z_{\mathrm{H}}=\frac{Z_{\mathrm{H}}-R_{\mathrm{H}}}{R_{\mathrm{H}}},
$$

where $R_{H}$ is the quantized Hall resistance, and $Z_{H}$ is the apparent two terminal-pair (2TP) impedance as seen by a measuring instrument at the extremes of the connections outside the cryostat.

The prediction of the model is

$$
\delta Z_{\mathrm{H}} \approx\left[\frac{Z_{\mathrm{w}} Y_{\mathrm{w}}}{2}+\left(\frac{R_{\mathrm{c}}+R_{\mathrm{w}}}{R_{\mathrm{H}}}\right)^{n}\right]^{2} .
$$

The quantities $Z_{\mathrm{w}}$ and $Y_{\mathrm{w}}$ can be estimated from the cable manufacturer specifications: in the following, the calculation is done for a $1.7 \mathrm{~m}$ long Lakeshore Ultra Miniature Coaxial Cable and a $0.3 \mathrm{~m}$ long RG58 coaxial cable.

The quantity $R_{C}$ is strongly dependent on the individual device employed in the experiment, and to some extent it can also vary for the same device in different cooling processes. We therefore computed the outcome of Equation 2 for two extreme cases, namely $R_{C}=10 \mathrm{~m} \Omega$ and $R_{C}=10 \Omega$.

Table 1 summarizes the result of the calculations performed at the frequency $f=1541 \mathrm{~Hz}$, which is of particular interest for the realisation of the farad unit from the quantized resistance [16]. It can be appreciated that, even for the case of a high contact resistance, a triple-series connection reduces the measurement error to about one part in $10^{8}$. The triple-series connection will be thus employed in the experiment. The residual error can be corrected if accurate measurements of the stray parameters are available.

\section{CONCLUSIONS}

The test shows that the probe can be employed to reach the quantization condition in Hall devices, and sensitive DC measurements can be performed.
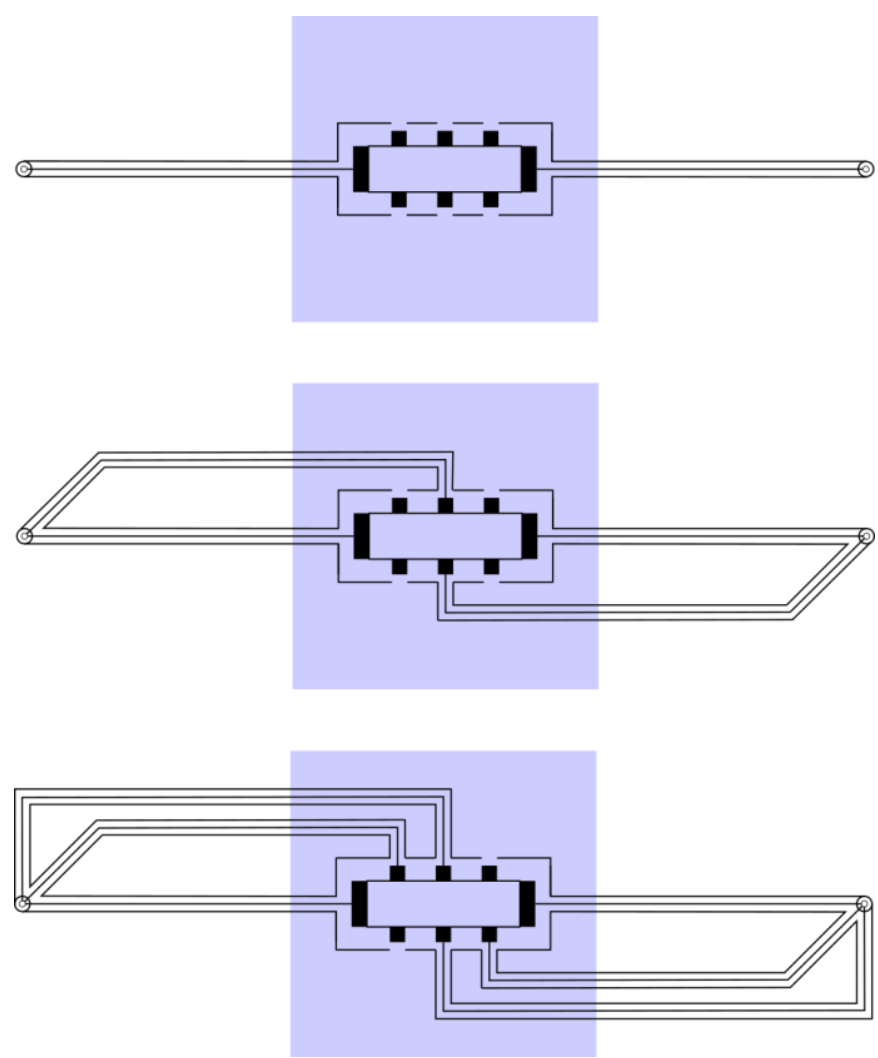

Figure 9. $n$-series connection of a QHE device. (top) $n=1$, no multiple-series connection: the device is connected as a 2TP impedance by two coaxial leads. (middle) $n=2$, double-series connection. (low) $n=3$, triple-series connection.

The probe is ready to be employed with a fully-digital coaxial impedance bridge designed for the calibration of a capacitance standard in terms of $R_{H}$ with an uncertainty of a few parts in $10^{7}$. In combination with the probe, the bridge is therefore suitable for the realisation of the unit of capacitance, the farad. The effects of stray parameters will be minimized by exploiting the triple-series connection technique, which reduces the connection errors to around one part in $10^{8}$.

The probe is intended to be used with graphene single devices and arrays [29], to be developed in the frame of the GIQS project. In the meantime, the GaAs device here investigated will allow to perform first tests of the bridge in operating conditions.

Measurements in the AC regime will be performed with a fully-digital bridge [16], using the triple-connection series; the approximate electrical modelling of the connections reported in the paper predicts a maximum connection error of a few parts in $10^{8}$. A more accurate modelling of such error, in progress, will allow to perform a correction of the measurement reading.

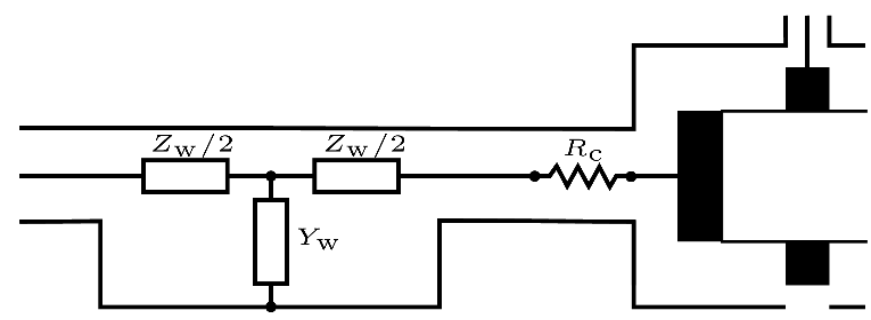

Figure 10 Electrical modelling of one of the connections to the QHE device. In the AC regime, each connection is modelled as a T network with series impedance $Z_{w}$ and a parallel admittance $Y_{w}$. The contact resistance $R c$ models the bonding and the device junctions. 


\section{ACKNOWLEDGEMENT}

GIQS: Graphene Impedance Quantum Standard is a Joint Research Project, code 18SIB07, of the European Metrology Programme for Innovation and Research (EMPIR). This project received funding from the European Metrology Programme for Innovation and Research (EMPIR) co-financed by the Participating States and from the European Union's Horizon 2020 research and innovation programme. Regular updates about the project are posted on the project webpage, ptb.de/empir2019/giqs/home/ and a LinkedIn group, linkedin.com/groups/8824119.

LC and VdE thank Cristina Cassiago and Enrico Gasparotto, INRIM, for helping with a first functionality test of the cryomagnet at the time of purchase.

\section{REFERENCES}

[1] Bureau International des Poids et Mesures, SI brochure, 9th edition, 2019. Online [Accessed 30 May 2021]

https://www.bipm.org

[2] R. Ribeiro-Palau, F. Lafont, J. Brun-Picard, D. Kazazis, A Michon, F. Cheynis, O. Couturaud, C. Consejo, B. Jouault, W. Poirier, F. Schopfer, Quantum Hall resistance standard in graphene devices under relaxed experimental conditions, Nature Nanotech 10 (2015), pp. 965-971.

DOI: $10.1038 /$ nnano.2015.192

[3] F. Lüönd, C.-C. Kalmbach, F. Overney, J. Schurr, B. Jeanneret, A Müller, M. Kruskopf, K. Pierz, F. Ahlers, AC quantum Hall effect in epitaxial graphene, IEEE Trans. Instrum. Meas. 66(6) (2017), pp. $1459-1466$.

DOI: $10.1109 /$ TIM.2017.2652501

[4] M. Kruskopf, R. E. Elmquist, Epitaxial graphene for quantum resistance metrology, Metrologia 55(4) (2018), pp. R27-R36. DOI: $10.1088 / 1681-7575 / \operatorname{aacd} 23$

[5] A. F. Rigosi, A. R. Panna, S. U. Payagala, M. Kruskopf, M. E. Kraft, G. R. Jones, B. Wu, H. Lee, Y. Yang, J. Hu, D. G. Jarrett, D. B. Newell, R. E. Elmquist, Graphene devices for tabletop and high-current quantized Hall resistance standards, IEEE Trans. Instr. Meas. 68(6) (2019), pp. 1870-1878.

DOI: $10.1109 /$ TIM.2018.2882958

[6] M. E. Cage, S. H. Shields, A. Jeffery, Initial NIST AC QHR measurements, J. Res. Natl. Inst. Stand. Technol. 109(4) (2004), pp. 391-405. Online [Accessed 10 June 2021]

https://nvlpubs.nist.gov/nistpubs/ires/109/4/i94cag.pdf

[7] F. J. Ahlers, B. Jeanneret, F. Overney, J. Schurr, B. M. Wood, Compendium for precise ac measurements of the quantum Hall resistance, Metrologia 46(5) (2009), pp. R1-R11.

DOI: $\frac{10.1088 / 0026-1394 / 46 / 5 / R 01}{2}$

[8] J. Kucera, P. Svoboda, K. Pierz, AC and DC quantum Hall measurements in GaAs-based devices at temperatures up to $4.2 \mathrm{~K}$, IEEE Trans. Instrum. Meas. 68(6) (2019), pp. 2106-2112. DOI: $10.1109 /$ TIM.2018.2882216

[9] J. Schurr, F. J. Ahlers, G. Hein, K. Pierz, The ac quantum Hall effect as a primary standard of impedance, Metrologia 44(1) (2007), pp. 15-23.

DOI: $10.1088 / 0026-1394 / 44 / 1 / 002$

[10] J. Schurr, V. Bürkel, B. P. Kibble, Realizing the farad from two ac quantum Hall resistances, Metrologia 46, 6 (2009), pp. 619-628. DOI: $10.1088 / 0026-1394 / 46 / 6 / 003$

[11] J. Schurr, J. Kucera, K. Pierz, B. P. Kibble, The quantum Hall impedance standard, Metrologia 48(1) (2011), pp. 47-57. DOI: $10.1088 / 0026-1394 / 48 / 1 / 005$

[12] L. Callegaro, Traceable measurements of electrical impedance, IEEE Instr. Meas. Mag. 18(6) (2015), pp. 42-46.

DOI: $10.1109 /$ MIM.2015.7335839
[13] F. Overney, B. Jeanneret, Impedance bridges: from Wheatstone to Josephson, Metrologia 55(5) (2018), pp. S119-S134. DOI: $10.1088 / 1681-7575 /$ aacf6c

[14] L. Callegaro, V. D’Elia, M. Kampik, D. B. Kim, M. Ortolano, F. Pourdanesh, Experiences with a two-terminal-pair digital impedance bridge, IEEE Trans. Instrum. Meas. 64(6) (2015), pp. $1460-1465$.

DOI: $10.1109 /$ TIM.2015.2401192

[15] J. Kucera, J. Kovác, A reconfigurable four terminal-pair digitally assisted and fully digital impedance ratio bridge, IEEE Trans. Instr. Meas. 67(5) (2018), pp. 1199-1206. DOI: $\underline{10.1109 / T I M .2018 .2790538}$

[16] M. Marzano, M. Ortolano, V. D’Elia, A. Müller, L. Callegaro, A fully digital bridge towards the realization of the farad from the quantum Hall effect, Metrologia 58(1) (2021), art. 015002. DOI: $10.1088 / 1681-7575 / a b b a 86$

[17] B. P. Kibble, J. Schurr, A novel double-shielding technique for ac quantum Hall measurement, Metrologia 45(5) (2008), pp. L25L27. DOI: $\underline{10.1088 / 0026-1394 / 45 / 5 / \mathrm{N} 01}$

[18] K. Pierz, B. Schumacher, Fabrication of quantum Hall devices for low magnetic fields, IEEE Trans. Instr. Meas. 48(2) (1999), pp. 293-295. DOI: $10.1109 / 19.769586$

[19] F. Piquemal, G. Genevès, F. Delahaye, J. P.-. Andrè, J. N.-Patillon, P. Frijlink, Report on a joint BIPM-EUROMET project for the fabrication of QHE samples by the LEP, IEEE Trans. Instr. Meas. 42(2) (1993), pp. 264-268. DOI: $10.1109 / 19.278562$

[20] L. Callegaro, Electrical Impedance: Principles, Measurement, and Applications, ser. Series in Sensors, CRC Press: Taylor and Francis, Boca Raton, FL, USA, 2013, ISBN 9781138199439

[21] R. D. Cutkosky, Four-terminal-pair networks as precision admittance and impedance standards, IEEE Transactions on Communication and Electronics 83(70) (1964), pp. 19-22. DOI: $10.1109 /$ TCOME.1964.6539563

[22] B. W. Ricketts, J. R. Fiander, H. L. Johnson, G. W. Small, Fourport AC quantized Hall resistance measurements, IEEE Trans. Instr. Meas. 52(2) (2003), pp. 579-583. DOI: $10.1109 /$ TIM.2003.810016

[23] B. W. Ricketts, P. C. Kemeny, Quantum Hall effect devices as circuit elements, J. Phys. D: Appl. Phys. 21(3) (1988), art. 483. DOI: $10.1088 / 0022-3727 / 21 / 3 / 018$

[24] F. Delahaye, Series and parallel connection of multiterminal quantum Hall effect devices, J. Appl. Phys. 73(11) (1993), pp. 7914-7920. DOI: $10.1063 / 1.353944$

[25] M. Ortolano, L. Callegaro, Matrix method analysis of quantum Hall effect device connections, Metrologia 49(1) (2012). DOI: $10.1088 / 0026-1394 / 49 / 1 / 001$

[26] M. Ortolano, L. Callegaro, Circuit models and SPICE macromodels for quantum Hall effect devices, Meas. Sci. Technol. 26 (2015), art. 085018. DOI: $10.1088 / 0957-0233 / 26 / 8 / 085018$

[27] M. Marzano, T. Oe, M. Ortolano, L. Callegaro, N.-H. Kaneko, Error modelling of quantum Hall array resistance standards, Metrologia 55(2) (2018), pp. 167-174. DOI: $10.1088 / 1681-7575 / \mathrm{aaa} 5 \mathrm{c} 1$

[28] J. Schurr, F.-J. Ahlers, G. Hein, J. Melcher, K. Pierz, F. Overney, B. M. Wood, AC longitudinal and contact resistance measurements of quantum Hall devices, Metrologia 43(1) (2006), pp. 163-173. DOI: $10.1088 / 0026-1394 / 43 / 1 / 021$

[29] M. Kruskopf, A. F. Rigosi, A. R. Panna, D. K. Patel, H. Jin, M. Marzano, M. Berilla, D. B. Newell, R. E. Elmquist, Two-terminal and multiterminal designs for next-generation quantized Hall resistance standards: Contact material and geometry, IEEE Trans. Electron Dev. 66(9) (2019), pp. 3973-3977. DOI: $\underline{10.1109 / T E D .2019 .2926684}$ 